\title{
RADIOMARCAJE Y ESTUDIOS DE BIODISTRIBUCIÓN DE NANOPARTÍCULAS POLIMÉRICAS COMO ADYUVANTES PARA VACUNACIÓN OFTÁLMICA FRENTE A BRUCELOSIS
}

María Sánchez-Martínez ${ }^{1}$, Raquel Da Costa Martins², Gemma Quincoces ${ }^{1}$, Carlos Gamazo ${ }^{3}$, Carlos Caicedo ${ }^{4}$, Juan M. Irache 2 , Iván Peñuelas ${ }^{1,5}$

${ }^{1}$ Unidad de Radiofarmacia, Departamento de Medicina Nuclear, Clínica Universidad de Navarra, Pamplona, 31008, España

${ }^{2}$ Departamento de Farmacia y Tecnología Farmacéutica, Universidad de Navarra, Pamplona, 31008, España

${ }^{3}$ Departamento de Microbiología, Universidad de Navarra, Pamplona, 31008, España

${ }^{4}$ Departamento de Medicina Nuclear, Clínica Universidad de Navarra, Pamplona, 31008, España

${ }^{5}$ Unidad de Investigación Micro-PET, Centro de Investigación Médica Aplicada-Clínica Universidad de Navarra (CIMA-CUN), Pamplona, 31008, España

Este trabajo ha sido en parte financiado por la "Asociación de Amigos de la Universidad de Navarra”, “Fundación Caja Navarra” (“Nanotecnología y Medicamentos", ref. 10828), el Ministerio de Educación y Ciencia (PCT-090100-2007-27), Ministerio de Ciencia e Innovación (AGLT2004-07088, PPT-420000-2008-4) y el Ministerio de Sanidad y Consumo (IF0663617-2).

Los autores expresan que no hay conflicto de intereses al redactar el manuscrito.

\section{AGRADECIMIENTOS}

Este trabajo ha sido en parte financiado por la "Asociación de Amigos de la Universidad de Navarra", “Fundación Caja Navarra" ("Nanotecnología y Medicamentos", ref. 10828), el 
Ministerio de Educación y Ciencia (PCT-090100-2007-27), Ministerio de Ciencia e Innovación (AGLT2004-07088, PPT-420000-2008-4) y el Ministerio de Sanidad y Consumo (IF0663617-2).

También nos gustaría agradecer la ayuda técnica proporcionada durante la realización de este trabajo de I. Bilbao y M. Ecay, Unidad de Investigación Micro-PET, Centro de Investigación Médica Aplicada-Clínica Universidad de Navarra (CIMA-CUN), Pamplona, España.

Los autores expresan que no hay conflicto de intereses al redactar el manuscrito.

\section{Autor principal para correspondencia:}

Iván Peñuelas, PhD

Unidad de Radiofarmacia

Departamento de Medicina Nuclear

Clínica Universidad de Navarra

Av. Pío XII, 36

31008 Pamplona, España

Tel.: + 34948255400 (ext.4943)

Fax: +34 948296500

ipenuelas@unav.es 


\section{RADIOMARCAJE Y ESTUDIOS DE BIODISTRIBUCIÓN DE NANOPARTÍCULAS POLIMÉRICAS COMO ADYUVANTES PARA VACUNACIÓN OFTÁLMICA FRENTE A BRUCELOSIS}

\section{RESUMEN}

Objetivos: Optimizar el radiomarcaje con ${ }^{99 \mathrm{~m}} \mathrm{Tc}$ de nanopartículas de $\mathrm{Gantrez}^{\circledR}$ manosiladas y cargadas con el antígeno de Brucella Ovis (Man-NP-HS) y llevar a cabo estudios de biodistribución en ratón tras la administración de las nanopartículas por vía ocular.

Metodología: Las Man-NP-HS se obtuvieron por el método de desplazamiento de disolvente. Se purificaron, liofilizaron y caracterizaron. A continuación, se marcaron con $74 \mathrm{MBq}$ de ${ }^{99 m} \mathrm{TcO}_{4}{ }^{-}$ previamente reducido con una disolución ácida de cloruro de estaño, trabajando en ausencia de oxígeno y con un $\mathrm{pH}$ final de 4. El rendimiento del marcaje se evaluó mediante TLC. Los estudios de biodistribución se llevaron a cabo en ratones tras la administración oftálmica de la formulación y de un control de ${ }^{99 m} \mathrm{TcO}_{4}{ }^{-}$libre. Para ello, se sacrificaron los animales a las 2 y a las 24 horas tras la administración ocular y se contaron los órganos en un contador gamma.

Resultados: Se obtuvo un rendimiento de marcaje superior al 90\%. Los estudios de biodistribución de ${ }^{99 m}$ Tc-Man-NP-HS permitieron detectar la actividad concentrada en mucosa nasal y ocular y tracto gastrointestinal tanto a las 2 como a las 24 horas, frente a la biodistribución de ${ }^{99 m} \mathrm{TcO}_{4}{ }^{-}$libre que permaneció concentrado en la piel alrededor del ojo y en tracto gastrointestinal.

Conclusión: Los estudios de biodistribución de ${ }^{99 m}$ Tc-Man-NP-HS tras administración oftálmica han permitido demostrar su biodistribución en mucosas y tracto gastrointestinal, característica indispensable como sistema de liberación de antígenos a través de mucosa ocular. Esto, junto con su elevada respuesta inmune, efectiva protección y no virulencia, convierte a estas nanopartículas en una vacuna ideal anti Brucelosis.

PALABRAS CLAVE: Nanopartículas; Biodistribución; Brucelosis; Vacunación ocular 
RADIOLABELLING AND BIODISTRIBUTION STUDIES OF POLYMERIC AS ADYUVANTS FOR ORAL VACCINATION AGAINST

\section{ABSTRACT}

Purpose: To optimize the radiolabelling with ${ }^{99 \mathrm{~m}} \mathrm{Tc}$ of Gantrez ${ }^{\circledR}$ mannosylated nanoparticles loaded with the Brucella Ovis antigen (Man-NP-HS) and carry out biodistribution studies in mice after the ocular administration of the nanoparticles.

Material and Methods: Man-NP-HS nanoparticles were prepared by the solvent displacement method. They were purified, lyophilized and characterized. Then, they were radiolabelled with $74 \mathrm{MBq}$ of ${ }^{99 m} \mathrm{TcO}_{4}{ }^{-}$previously reduced with an acidic stannous chloride solution, working in absence of oxygen and at a final $\mathrm{pH}$ of 4 . Radiolabelling yield was evaluated by TLC. Biodistribution studies were carried out in mice after ocular administration of the formulation and a control of free ${ }^{99 \mathrm{~m}} \mathrm{TcO}_{4}^{-}$. Thus, animals were humanly killed at 2 and 24 hours after the administration and activity in organs was measured in a Gamma counter.

Results: The radiolabelling yield obtained was more than $90 \%$. Biodistribution studies of ${ }^{99 m}$ Tc-Man-NPHS showed radioactivity accumulated at 2 and 24 hours in nasal and ocular mucosa and gastrointestinal tract, in contrast to biodistribution of free ${ }^{99 \mathrm{~m}} \mathrm{TcO}_{4}{ }^{-}$, that remained concentrated in the skin around the eye and gastrointestinal tract.

Conclusion: Biodistribution studies of ${ }^{99 m}$ Tc-Man-NP-HS revealed that, after ocular instillation, nanoparticles were distributed to the nasal mucosa and gastrointestinal tract thus permitting an increase of the mucosal immune response. These characteristics make them an excellent antigenic delivery system throughout the ocular mucosa. Besides, their effective protection and intrinsic avirulence, make them a suitable anti-Brucella vaccine candidate.

KEY WORDS: Nanoparticles; Biodistribution; Brucellosis; Ocular vaccination 


\section{INTRODUCCIÓN}

La brucelosis es una de las zoonosis bacterianas más contagiosas del mundo ${ }^{1}$ y afecta tanto al ganado doméstico como a algunos animales salvajes ${ }^{2,3}$. Se transmite al ser humano por contacto directo con ganado infectado o por consumo de productos lácteos no higienizados, causando síntomas crónicos graves e invalidantes cuyo tratamiento es largo y costoso ${ }^{4}$. En el ganado causa abortos e infertilidad y cuantiosas pérdidas económicas. Sólo los países no mediterráneos de la Unión Europea, Canadá, Estados Unidos, Japón, Australia y Nueva Zelanda han erradicado esta enfermedad de los rumiantes tras décadas de vacunación y costosas medidas de sacrificio de animales.

No existen vacunas humanas contra brucelosis y las vacunas animales existentes, Brucella melitensis Rev1 y Brucella abortus RB51, presentan grandes inconvenientes debido a que se basan en la bacteria viva atenuada ${ }^{5,6,7,8}$ por lo que surge la necesidad del desarrollo de nuevas vacunas más seguras y eficaces.

Para superar dichos inconvenientes, la tendencia actual se orienta hacia el empleo de vacunas subcelulares. Se ha demostrado que el extracto antigénico subcelular Hot Saline (HS) de Brucella ovis presenta una elevada inmunogenicidad ${ }^{9,10}$. Sin embargo, debido a la naturaleza no replicante del antígeno se requiere el empleo de adyuvantes adecuados, tales como nanopartículas poliméricas biodegradables y bioadhesivas que incrementen la respuesta inmune. Estas nanopartículas también permiten la administración de la vacuna por vías alternativas a las parenterales, como son las vías mucosales, y dentro de éstas, la administración ocular se ha revelado como una de las vías más económicas para conseguir una mayor y más rápida biodisponibilidad de fármacos ${ }^{5}$. 
Las nanopartículas de metil vinil éter y anhídrido maléico (preparadas con Gantrez ${ }^{\circledR}$ AN 119) cuando son utilizadas como adyuvantes mucosales incrementan la estabilidad del antígeno HS y lo protegen frente a la degradación, aumentan su biodisponibilidad por esta vía, imitan la vía de entrada del patógeno y favorecen la respuesta inmune $e^{11,12,13,14}$. A su vez, la incorporación de manosamina sobre la superficie de las nanopartículas desarrolla interacciones específicas con receptores expresados en células del sistema inmune aumentando la respuesta inmunitaria innata $^{15,16}$.

Una limitación sustancial de numerosos estudios previos es la dificultad de detectar la biodistribución de dichas formulaciones de nanopartículas tras su administración vía mucosas. El empleo de técnicas basadas en el uso de trazadores radioisotópicos permite realizar estudios de biodistribución in vivo y ex vivo. Debido a las adecuadas propiedades físicas del ${ }^{99 \mathrm{~m}} \mathrm{Tc}\left(\mathrm{T}_{1 / 2}=6,02\right.$ horas, emisor gamma puro de $\left.140 \mathrm{keV}\right)$ y a su gran disponibilidad mediante un generador de ${ }^{99} \mathrm{Mo}-{ }^{99 \mathrm{~m}} \mathrm{Tc}$, lo convierten en el isótopo de elección para el radiomarcaje de las nanopartículas, lo cual permitirá ver la localización en los diferentes órganos del animal inoculado en tiempos de hasta 72 horas tras la administración oftálmica.

Por lo tanto, el próposito de este estudio es optimizar el radiomarcaje con tecnecio-99m de nanopartículas de Gantrez ${ }^{\circledR}$ manosiladas y cargadas con el antígeno de Brucella Ovis (Man-NPHS) para poder así llevar a cabo estudios de biodistribución en ratón tras la administración de las nanopartículas marcadas por vía ocular como una forma adicional de obtener información que pudiera explicar el efecto inmunogénico de las nanopartículas administradas.

MATERIAL Y MÉTODOS

Reactivos 
El copolímero de metil vinil éter y anhídrido maléico o polianhídrido (Gantrez AN 119; Peso molecular $200 \mathrm{kDa}$ ) fue suministrado por ISP (Barcelona, España). El generador de ${ }^{99} \mathrm{Mo}-{ }^{99 \mathrm{~m}} \mathrm{Tc}$ (Drytec; GE Healthcare Bio-science, Reino Unido) se eluyó con $\mathrm{NaCl}$ al 0,9 \% siguiendo las instrucciones del fabricante. $\mathrm{El} \mathrm{NaCl}$ al 0,9 \% y el agua para inyectables se obtuvieron de Braun Medical S.A. (Barcelona, España). La metil etil cetona, el acetato sódico anhidro, la acetona, el etanol, el cloruro de estaño dihidrato y el ácido clorhídrico fueron suministros por Panreac (Barcelona, España). Todos los reactivos usados en este estudio fueron de calidad analítica.

\section{Animales}

Se utilizaron 32 ratones hembra Balb/c de ocho semanas de edad con un peso medio de $20 \pm 1$ gramos que fueron cuidados y alimentados bajo condiciones estándar con libre acceso a comida y bebida. Los ratones se trasladaron a jaulas metabólicas 12 horas antes de empezar el estudio para prevenir la coprofagia (la cual alteraría el estudio) pero permitiéndoles libre acceso al agua. Todos los procedimientos se llevaron a cabo siguiendo un protocolo previamente aprobado por el Comité ético de la Universidad de Navarra en línea con la legislación europea de animales de experimentación (86/609/EU).

\section{Preparación de las nanopartículas poliméricas}

Las nanopartículas poliméricas manosiladas y funcionalizadas con el extracto antigénico (ManNP-HS), las nanopartículas poliméricas funcionalizadas con el extracto antigénico (NP-HS) y sus respectivos controles (Man-NP y NP) se prepararon por una modificación del método de desolvatación en doble disolvente descrito previamente ${ }^{17,18}$. Para la preparación de Man-NP 
HS, 100 mg de Gantrez ${ }^{\circledR}$ AN 119 se disolvieron en $5 \mathrm{ml}$ de acetona y se incubaron durante 8 horas con $1 \mathrm{mg}$ de D-manosamina. Posteriormente, se añadieron $4 \mathrm{mg}$ del extracto de antígeno hot saline (HS) obtenidos por el método previamente descrito ${ }^{10}$ y se sonicó la muestra durante 10 minutos. Tras 30 minutos de incubación bajo agitación magnética a temperatura ambiente y la adición de una mezcla de etanol/agua (1:1) se produjo la formación de las nanopartículas, que seguidamente se dispersaron en $10 \mathrm{ml}$ de agua ultrapura que contenía $5 \mathrm{mg}$ de manosamina. A continuación, se eliminaron los disolventes orgánicos mediante evaporación bajo presión reducida en un rotavapor (Buchi, R-144, Suiza) y la nanosuspensión acuosa obtenida se mantuvo una hora bajo agitación magnética con el fin de facilitar el recubrimiento de las nanopartículas con manosamina. Tras la incubación, las nanopartículas se purificaron mediante centrifugación a 3.000 g durante 20 minutos (VivaSpin ${ }^{\circledR} 20$ 300,000 M.W.C.O., Vivascience, Sartorius group, Hannover, Alemania). Los sobrenadantes se extrajeron y se utilizaron para cuantificar la cantidad de HS no atrapado y manosamina no asociada y los pellets se resuspendieron en agua. El proceso de purificación se repitió dos veces y finalmente las formulaciones se congelaron y liofilizaron (Genesis 12 EL; Virtis, EE.UU.) utilizando sacarosa ( $5 \%$ peso/peso) como crioprotector. Las nanopartículas poliméricas funcionalizadas con el extracto antigénico (NP-HS) se prepararon siguiendo el mismo procedimiento pero en ausencia de manosamina, las nanopartículas manosiladas control (Man-NP) se prepararon en ausencia del antígeno HS y las nanopartículas control (NP) en ausencia del antígeno HS y manosamina. En la figura 1a se esquematiza este proceso de preparación. Las nanopartículas se caracterizaron mediante la medida de su tamaño, potencial zeta y morfología siguiendo métodos previamente descritos ${ }^{19}$. 
Radiomarcaje de nanopartículas con ${ }^{99 m} \mathrm{Tc}$

El radiomarcaje de las nanopartículas se llevó a cabo con ${ }^{99 m} \mathrm{Tc}$ por reducción de ${ }^{99 \mathrm{~m}} \mathrm{Tc}$ pertecnetato $\left({ }^{99 \mathrm{~m}} \mathrm{TcO}_{4}{ }^{-}\right)$con cloruro de estaño siguiendo el método previamente descrito ${ }^{20}$. Para ello, $20 \mu \mathrm{l}$ de una disolución de cloruro de estaño dihidrato con pH ajustado a 1 con una disolución de ácido clorhídrico $0.1 \mathrm{~N}$ y una concentración final de estaño de $0.03 \mathrm{mg} / \mathrm{ml}$ se añadió a 1 mg de las formulaciones de nanopartículas liofilizadas (tanto NP como NP-HS, ManNP y Man-NP-HS) seguido de la adición de $74 \mathrm{MBq}$ de eluido de ${ }^{99 \mathrm{~m}} \mathrm{TcO}_{4}^{-}$en $0.5 \mathrm{ml}$ de $\mathrm{NaCl}$ al 0.9\% añadidos sobre el estaño prerreducido. A continuación se agitó la mezcla en un vórtex durante 30 segundos y se incubó a temperatura ambiente durante 10 minutos. El pH de la suspensión resultante fue de 4. Todo el proceso se llevó a cabo en viales purgados con helio y se utilizaron disoluciones purgadas con helio para minimizar la cantidad de oxígeno presente y evitar la oxidación del estaño prerreducido. En la figura 1b se puede ver un esquema del proceso de radiomarcaje.

\section{Control de calidad de las nanopartículas radiomarcadas}

Las distintas suspensiones de ${ }^{99 \mathrm{~m}} \mathrm{Tc}$-nanopartículas se analizaron mediante un sistema de cromatografía en capa fina (TLC) con tiras de silicagel (Polygram ${ }^{\circledR}$ sil N-HR; Macherey-Nagel, Düren, Alemania) utilizando un método de doble disolvente ${ }^{21}$. Para ello, una microgota de la muestra se añadió a $1 \mathrm{~cm}$ de la tira y se desarrolló hasta $9 \mathrm{~cm}$ con metiletil cetona (MEK). A continuación, se dejó secar la tira al aire y se desarrolló nuevamente con una disolución acuosa de acetato sódico al $18 \%(\mathrm{p} / \mathrm{v})$ hasta $4.5 \mathrm{~cm}$. Las tiras de ITLC se leyeron en un 
radiocromatógrafo MiniGita equipado con un detector $2 \times 2$ de Nal(TI) y el software Nina (Raytest GmbH, Dortmund, Alemania).

\section{Estudios de biodistribución ex vivo con nanopartículas radiomarcadas}

Los estudios de biodistribución ex vivo se llevaron a cabo en ratones hembra Balb/c. Se establecieron cinco grupos de 6 ratones cada uno $\left({ }^{99 \mathrm{~m}} \mathrm{Tc}-\mathrm{NP},{ }^{99 \mathrm{~m}} \mathrm{Tc}-\right.$ Man-NP, ${ }^{99 \mathrm{~m}} \mathrm{Tc}-\mathrm{NP}-\mathrm{HS},{ }^{99 \mathrm{~m}} \mathrm{Tc}-$ Man-NP-HS y ${ }^{99 m} \mathrm{TcO}_{4}{ }^{-}$libre). Una hora antes de la administración de las distintas formulaciones se inyectó a los ratones una dosis intravenosa de perclorato potásico de $3 \mathrm{mg} / \mathrm{Kg}$ para bloquear la captación en estómago y evitar así errores en los resultados debidos a la captación en estómago y tiroides de la pequeña proporción de ${ }^{99 \mathrm{~m}} \mathrm{TcO}_{4}{ }^{-}$libre que pudiera haber en la formulación ${ }^{22}$. Una única dosis de $8 \mu$ lde cada formulación se depositó en cada ojo de los ratones de cada grupo. Los animales se sacrificaron por dislocación cervical 2 o 24 horas después de la administración ocular de las formulaciones. A continuación, los animales fueron diseccionados y se extrajeron y pesaron sus órganos para su posterior contaje en un contador gamma (Compugamma CS, RIA; LKB Pharmacia, Finlandia). Finalmente, los resultados se expresaron como el porcentaje de cuentas por minuto por gramo de cada órgano dividido por las cuentas por minuto totales dividido por el peso total de todos los órganos ((cpm/gr)/(cpm total/gr total)).

Además, transcurridas 4 horas de la administración ocular de la formulación ${ }^{99 m}$ Tc-Man-NP-HS en un animal se adquirió durante 1 hora una imagen planar de los órganos más representativos con un equipo SPECT-TAC de doble cabezal (SYMBIA-T2 TruePoint ${ }^{\circledR}$; Siemens, Munich, Alemania) y con una matriz de 128 x 128 y zoom de 1.23. 
Estudios de biodistribución con nanopartículas radiomarcadas mediante autorradiografía digital semicuantitativa y fluorescencia

Los estudios de biodistribución in vivo se llevaron a cabo en ratones Balb/c que permanecieron 12 horas en ayunas. Una hora antes de la administración se administró a los ratones una dosis intravenosa de perclorato potásico de $3 \mathrm{mg} / \mathrm{Kg}$. Una única dosis de $8 \mu \mathrm{l}$ de la formulación ${ }^{99 m}$ Tc-Man-NP-HS (2,37 MBq, 12 g de HS por ojo) se depositó sobre los ojos de los ratones. Cuatro horas después de la administración los animales fueron anestesiados con un $2 \%$ de isofluorano y posteriormente eutanizados con T-61, congelados en nitrógeno líquido, tratados con el medio de procesamiento de tejido O.C.T. (Tissue-Tek, Sakura, Países Bajos) y almacenados a $-20^{\circ} \mathrm{C}$. A continuación, se procedió a realizar cortes longitudinales de los animales de $20 \mu \mathrm{m}$ de sección con un criostato (8250 Bright Instrument CoLtd, Cambridge shire, Inglaterra) y se colocaron en placas de imagen (BAS-SR 2025, Fujifilm, Japón) durante 20 minutos. Después, se revelaron las placas con un equipo de autorradiografía digital semicuantitativa (BAS-500, Fujifilm, Japón) y las imágenes se trataron con el software Raytest BAS500 reader 3.14.

\section{RESULTADOS}

Radiomarcaje con ${ }^{99 m} T c$ y control de calidad de las nanopartículas marcadas

La eficacia del radiomarcaje se estudió mediante cromatografía en capa fina, TLC. Para ello, se empleó un método basado en el uso de una única tira de cromatografía y dos disolventes, MEK y acetato sódico al $18 \%$. Utilizando este sistema es posible separar claramente los tres 
componentes de la muestra: ${ }^{99 m} \mathrm{Tc}$-coloides $\left(R_{f}=0\right),{ }^{99 m} \mathrm{TcO}_{4}{ }^{-}$libre $\left(R_{f}=1\right)$ y ${ }^{99 m} T c-N P\left(R_{f} \geq 0.5\right)$ y se obtuvo un rendimiento de marcaje superior al $90 \%$.

\section{Cuantificación ex vivo}

En la figura 2 se muestran los resultados de biodistribución de cada órgano/tejido tras la administración oftálmica de las nanopartículas marcadas ${ }^{99 \mathrm{~m}} \mathrm{Tc}-\mathrm{Man}-\mathrm{NP}$-HS en donde se puede observar que la mayor acumulación de radiactividad, tanto a las 2 como a las 24 horas tras la administración ocular, se encontró tanto en mucosa nasal $(6 \% \pm 0.15$ a las 2 horas, $13 \% \pm 1.22$ a las 24 horas $)$ como ocular $(44 \% \pm 2.50$ a las 2 horas, $8 \% \pm 0.30$ a las 24 horas $)$ y en tracto gastrointestinal $(47 \% \pm 2.94$ a las 2 horas, $70 \% \pm 0.43$ a las 24 horas). También se pudo apreciar que parte de la actividad permanecía adherida en la piel alrededor de los ojos, pero en una pequeña proporción ( $15 \% \pm 1.31$ a las 2 horas, $9 \% \pm 0.30$ a las 24 horas). En cambio, se observó que la mayor acumulación de actividad de ${ }^{99 \mathrm{~m}} \mathrm{TcO}_{4}{ }^{-}$libre permanecía en la piel alrededor de los ojos tras las administración ( $68 \% \pm 0.66$ a las 2 horas, $74 \% \pm 0.49$ a las 24 horas). También se pudo ver que de la actividad que penetra en el cuerpo del animal, la mayor parte se distribuía en tracto gastrointestinal ( $12 \% \pm 0.50$ a las 2 horas, $21 \% \pm 0.29$ a las 24 horas).

Los estudios de biodistribución de todas las formulaciones, ${ }^{99 \mathrm{~m}} \mathrm{Tc}-\mathrm{Man}-\mathrm{NP}-\mathrm{HS},{ }^{99 \mathrm{~m}} \mathrm{Tc}-\mathrm{NP},{ }^{99 \mathrm{~m}} \mathrm{Tc}-$ Man-NP y ${ }^{99 m}$ Tc-NP-HS, mostraron una acumulación de actividad similar.

En la figura 3 se muestra una imagen planar de gammacámara correspondiente con la localización de las nanopartículas marcadas ${ }^{99 \mathrm{~m}} \mathrm{Tc}$-Man-NP-HS en los distintos órganos de los animales después del sacrificio a las 4 horas. Como era de esperar, la radiactividad se observó en la mucosa ocular y nasal, en la piel alrededor de los ojos y en el tracto gastrointestinal, 
corroborando los resultados obtenidos mediante las medidas de actividad en el contador Gamma.

Estudios de autorradiografía.

En la figura 4 se muestra una imagen de autorradiografía en la que se puede observar la biodistribución de las nanopartículas marcadas ${ }^{99 \mathrm{~m}} \mathrm{Tc}-\mathrm{Man}-\mathrm{NP}-\mathrm{HS}$ transcurridas 4 horas desde su administración oftálmica. Mediante esta técnica se observó que se corroboraban también los resultados obtenidos mediante los estudios de biodistribución ex vivo, es decir, la radiactividad se distribuyó en la mucosa ocular, en la mucosa nasal y en el tracto gastrointestinal.

\section{DISCUSIÓN}

Numerosas publicaciones hablan de las propiedades de las nanopartículas poliméricas de Gantrez $^{\circledR}$ AN 119 como adyuvantes vacunales vía mucosal ${ }^{23,24,25}$ sin embargo, poco se conoce acerca de su comportamiento y biodistribución in vivo. Para superar estas limitaciones, en el presente estudio se han marcado dichas nanopartículas con el isótopo radiactivo ${ }^{99 \mathrm{~m}} \mathrm{Tc}$ con el objetivo de evaluar la distribución de nanopartículas poliméricas de Gantrez ${ }^{\circledR}$ AN 119 manosiladas y cargadas con el antígeno subcelular Hot Saline de Brucella ovis (Man-NP-HS) tras su administración oftálmica en ratón.

Durante años, en la búsqueda de fármacos para el tratamiento de enfermedades oculares mediante gotas se ha intentado que dichos fármacos superen la barrera del epitelio corneal 
penetrando así en la cornea y alcanzando los tejidos intraoculares y se ha buscado evitar en lo posible la absorción del fármaco y penetración en el organismo mediante la circulación sistémica $^{26}$. Otro requisito a tener en cuenta ha sido evitar la pérdida de fármaco por medio de lacrimación y aumentar el tiempo de residencia del fármaco en el tejido corneal para favorecer su absorción. En nuestro caso, al tratarse de una vacuna anti Brucelosis nos interesa que el fármaco penetre también en el organismo para que se activen los tejidos linfoides, también presentes en la conjuntiva y en el sistema de drenaje |acrimal ${ }^{27}$. Esto se refleja en los resultados obtenidos de los estudios isotópicos de biodistribución ex vivo mediante contaje de órganos (Figura 2) que mostraron que tanto a las 2 como a las 24 horas la mayor parte de la radiactividad se acumulaba en mucosa ocular y nasal y en tracto gastrointestinal. Esto se debe a que las nanopartículas, que presentan gran capacidad de adhesión a mucosas, una vez que entran al organismo por vía ocular se distribuyen también por el resto de mucosas, como son la mucosa nasal y la mucosa del tracto gastrointestinal. A su vez, el hecho de que a las 24 horas siga habiendo acumulación de las nanopartículas radiomarcadas en los mismos tejidos/órganos nos indica la alta bioadhesion que presentan estos adyuvantes con las mucosas, con lo que se produce un aumento del tiempo de residencia de la formulación en el organismo, lo que permite una mayor biodisponibilidad del antígeno y por tanto una mayor activación del sistema inmune. En su preparación las nanopartículas fueron además recubiertas con el ligando manosamina para favorecer las interacciones con receptores específicos del sistema inmune ${ }^{15,16}$ mejorando así todavía más la respuesta inmunitaria.

Estos resultados contrastan con los obtenidos tras la administración de ${ }^{99 \mathrm{~m}} \mathrm{TcO}_{4}^{-}$libre (Figura 2), que al no presentar propiedades bioadhesivas se desprende en su mayor parte del ojo en la administración. 
Distintas técnicas han permitido confirmar estos resultados, ya que a parte de los estudios ex vivo de contaje de órganos en un contador gamma se realizaron estudios de autoradiografía y gammagráficos (Figuras 3 y 4) que obtuvieron resultados concordantes. La importancia de estos resultados nos lleva a la posibilidad del uso de las técnicas de imagen molecular en estudios de biodistribución de fármacos y de farmacocinética, presentando la ventaja de ser técnicas no invasivas.

\section{CONCLUSIONES}

En este estudio se ha demostrado que es posible obtener un buen rendimiento en el marcaje con ${ }^{99 \mathrm{~m}} \mathrm{Tc}$ de nanopartículas poliméricas de Gantrez ${ }^{\circledR}$ AN 119 manosiladas y cargadas con el antígeno subcelular Hot Saline de Brucella ovis (Man-NP-HS). A su vez, los estudios de biodistribución en ratón tras la administración oftálmica de ${ }^{99 \mathrm{~m}} \mathrm{Tc}-\mathrm{Man}-\mathrm{NP}-\mathrm{HS}$ han demostrado que estas nanopartículas presentan una excelente distribución en mucosas y tracto gastrointestinal, lo que las convierte en un efectivo sistema de liberación de antígenos a través de mucosa ocular. Todo esto, junto con su demostrada elevada respuesta inmune, efectiva protección y no virulencia ${ }^{28,29,30}$ convierten a estas nanopartículas en una potencial vacuna ideal anti brucelosis.

\section{REFERENCIAS}

[1] Boschiroli ML, Foulongne V, O'Callaghan D. Brucellosis: a worldwide zoonosis. Curr Opin Microbiol 2001;4(1):58-64 
[2] Cutler, S.J., Whatmore, A.M., Commander, N.J., 2005. Brucellosis-new aspects of an old disease. J. Appl. Microbiol. 98, 1270-81.

[3] Whatmore, A.M., Dawson, C.E., Groussaud, P., Koylass, M.S., King, A.C., Shankster,S.J., et al, 2008. Marine mammal Brucella genotype associated with zoonotic infection. Emerg. Infect. Dis. $14,517-8$.

[4] Young, E.J., 1995. An overview of human brucellosis. Clin. Infect. Dis. 21, 283-9 (quiz 290).

[5] Blasco, J.M., 1997. A review of the use of B. melitensis Rev 1 vaccine in adult sheep and goats. Prev. Vet. Med. 31, 275-83.

[6] Blasco, J.M., Diaz, R., 1993. Brucella melitensis Rev-1 vaccine as a cause of human brucellosis. Lancet 342, 805 .

[7] Schurig, G.G., Sriranganathan, N., Corbel, M.J., 2002. Brucellosis vaccines: past, present and future. Vet. Microbiol. 90, 479496.

[8] Moriyon, I., Grillo, M.J., Monreal, D., Gonzalez, D., Marin, C., Lopez-Goni, I., et al, 2004. Rough vaccines in animal brucellosis: structural and genetic basis and present status. Vet. Res. $35,1-38$.

[9] Blasco, J.M., Gamazo, C., Winter, A.J., Jimenez de Bagues, M.P., Marin, C., Barberan, M., et al, 1993. Evaluation of whole cell and subcellular vaccines against Brucella ovis in rams. Vet. Immunol. Immunopathol. 37, 257-70.

[10] Gamazo, C.,Winter,A.J., Moriyon, I., Riezu-Boj, J.I., Blasco, J.M., Diaz, R., 1989.Comparative analyses of proteins extracted by hot saline or released spontaneously into outer membrane blebs from field strains of Brucella ovis and Brucella melitensis. Infect. Immun. 57, 1419-26. 
[11] Almeida, A.J., Alpar, H.O., 1996. Nasal delivery of vaccines. J. Drug Target. 3, 455-67.

[12] Arbos, P., Campanero, M.A., Arangoa, M.A., Renedo, M.J., Irache, J.M., 2003. Influence of the surface characteristics of PVM/MA nanoparticles on their bioadhesive properties. J. Control. Release 89, 19-30.

[13] Gomez, S., Gamazo, C., Roman, B.S., Ferrer, M., Sanz, M.L., Irache, J.M., 2007. Gantrez AN nanoparticles as an adjuvant for oral immunotherapy with allergens. Vaccine 25, 5263-71.

[14] Motwani, S.K., Chopra, S., Talegaonkar, S., Kohli, K., Ahmad, F.J., Khar, R.K., 2008. Chitosan-sodium alginate nanoparticles as submicroscopic reservoirs for ocular delivery: formulation, optimisation and in vitro characterisation. Eur. J. Pharm. Biopharm. 68, 513-25.

[15] Engering, A.J., Cella, M., Fluitsma, D., Brockhaus, M., Hoefsmit, E.C., Lanzavecchia, A., et al, 1997. The mannose receptor functions as a high capacity and broad specificity antigen receptor in human dendritic cells. Eur. J. Immunol. 27, 2417-25.

[16] Jain, S., Vyas, S.P., 2006. Mannosylated niosomes as adjuvant-carrier system for oral mucosal immunization. J. Liposome Res. 16, 331-45.

[17] Arbos, P., Wirth, M., Arangoa, M.A., Gabor, F., Irache, J.M., 2002. Gantrez AN as a new polymer for the preparation of ligand-nanoparticle conjugates. J. Control. Release 83, 321-30.

[18] Salman, H.H., Gamazo, C., Campanero, M.A., Irache, J.M., 2006. Bioadhesive mannosylated nanoparticles for oral drug delivery. J. Nanosci. Nanotechnol. 6, 3203-9.

[19] R. Da Costa Martins, C. Gamazo, J.M. Irache. Design and influence of gamma-irradiation on the biopharmaceutical properties of nanoparticles containing an antigenic complex from Brucella ovis, Eur. J. Pharm. Sci. 37 (2009) 563-72. 
[20] Agüeros M., Areses P,. Campanero M.A. et al. Bioadhesive properties and biodistribution of cyclodextrin-poly(anhydride) nanoparticles. Eur J Pharm Sci 2009; 37:231-40

[21] P. Areses, M. Agueros, G. Quincoces, M. Collantes, J.A. Richter, L.M. Lopez-Sanchez, et al. Molecular imaging techniques to study the biodistribution of orally administered $(99 \mathrm{~m})$ Tclabelled naive and ligand-tagged nanoparticles, Mol. Imaging Biol. 2011;13:1215-23.

[22] Clewell RA, Merrill EA, Narayanan L, Gearhart JM, Robinson PJ. Evidence for competitive inhibition of iodide uptake by perchlorate and translocation of perchlorate into the thyroid. Int J Toxicol. 2004 Jan-Feb;23(1):17-23.

[23] Ponchel G, Irache J. Specific and non-specific bioadhesive particulate systems for oral delivery to the gastrointestinal tract. Adv Drug Deliv Rev 1998;34:191-219.

[24] Arbos P, Wirth M, Arangoa MA, Gabor F, Irache JM. Gantrez AN as a new polymer for the preparation of ligand-nanoparticle conjugates. J Control Release 2002;83(3):321-30.

[25] Irache JM, Salman HH, Gomez S, Espuelas S, Gamazo C. Poly(anhydride) nanoparticles as adjuvants for mucosal vaccination. Front. Biosci. (Schol Ed). 2010 Jun 1;2:876-90.

[26] Järvinen K, Järvinen M, Urtti, A. Ocular absorption following topical delivery. Adv. Drug Del. Rev. 1995;16:3-19.

[27] M. de la Fuente, M. Raviña, P. Paolicelli, A. Sanchez, B. Seijo, M.J. Alonso. Chitosan-based nanostructures: A delivery platform for ocular therapeutics. Adv. Drug Del. Rev. 2010;62:10017. 
[28] R. Da Costa Martins, C. Gamazo, J.M. Irache. Design and influence of g-irradiation on the biopharmaceutical properties of nanoparticles containing an antigenic complex from Brucella ovis. Eur. J. Pharm. Sci. 2009;37:563-72.

[29] R. Da Costa Martins, J.M. Irache, J.M. Blasco, M.P. Munoz, C.M. Marin, M.J. Grillo, et al. Evaluation of particulate acellular vaccines against Brucella ovis infection in rams. Vaccine 2010;28:3038-46.

[30] R. Da Costa Martins, C. Gamazo, M. Sánchez-Martínez, M. Barberán, I. Peñuelas, J.M. Irache. Conjunctival vaccination against Brucella ovis in mice with mannosylated nanoparticles. J. Control.Release 2012;162:553-60. 
Figura 1. a) Preparación de las formulaciones de nanopartículas mediante el método de desolvatación en doble disolvente (etanol/agua). Tras la formación de las suspensiones de NP se procede a la eliminación de disolventes mediante presión reducida en un rotavapor, a la purificación mediante centrifugación y la posterior liofilización usando sacarosa como crioprotector. b) Reacción de radiomarcaje de Man-NP-HS con ${ }^{99 m} \mathrm{Tc}$. El proceso de reducción de ${ }^{99 \mathrm{~m}} \mathrm{TcO}_{4}{ }^{-}$obtenido del generador se lleva a cabo con $\mathrm{SnCl}_{2} \cdot 2 \mathrm{H}_{2} \mathrm{O}$ en medio ácido $(\mathrm{pH}=1)$ y trabajando en ausencia de oxígeno. El tiempo necesario para el marcaje es de 10 minutos, obteniéndose una suspención de NP de $\mathrm{pH}=4$. 
Figura 2. Estudios de biodistribución tras 2 horas (barras blancas) y 24 horas (barras negras) de la administración oftálmica de ${ }^{99 \mathrm{~m}} \mathrm{Tc}-\mathrm{Man}-\mathrm{NP}-\mathrm{HS}$ y de ${ }^{99 \mathrm{~m}} \mathrm{TcO}_{4}^{-}$libre tras 2 horas (barras rayadas) y 24 horas (barras punteadas). Los resultados muestran la media de tres animales. Se puede observar la distribución de ${ }^{99 \mathrm{~m}} \mathrm{Tc}-\mathrm{Man}-\mathrm{NP}-\mathrm{HS}$ principalmente en mucosas nasales y oculares y tracto gastrointestinal frente a la biodistribución de ${ }^{99 \mathrm{~m}} \mathrm{TcO}_{4}{ }^{-}$libre que permanece mayoritariamente en la piel alrededor de los ojos y en una pequeña proporción en tracto gastrointestinal. 
Figura 3. (a) Placa de 12 pocillos con los órganos de interés extraídos de un ratón transcurridas 4 horas de la administración oftálmica de ${ }^{99 m}$ Tc-Man-NP-HS. (b) Imagen planar de gammacámara de la placa anterior donde se puede observar la acumulación de la radiactividad en mucosa ocular, tracto gastrointestinal y en la piel que rodeada al ojo. 
Figura 4. Imágenes de Autorradiografía de dos cortes de un mismo animal a dos profundidas distintas transcurridas 4 horas de la administración oftálmica de ${ }^{99 m}$ Tc-Man-NP-HS. En ambos cortes se puede observar la biodistribución de la formulación en mucosas oculares y nasales y en tracto gastrointestinal. 

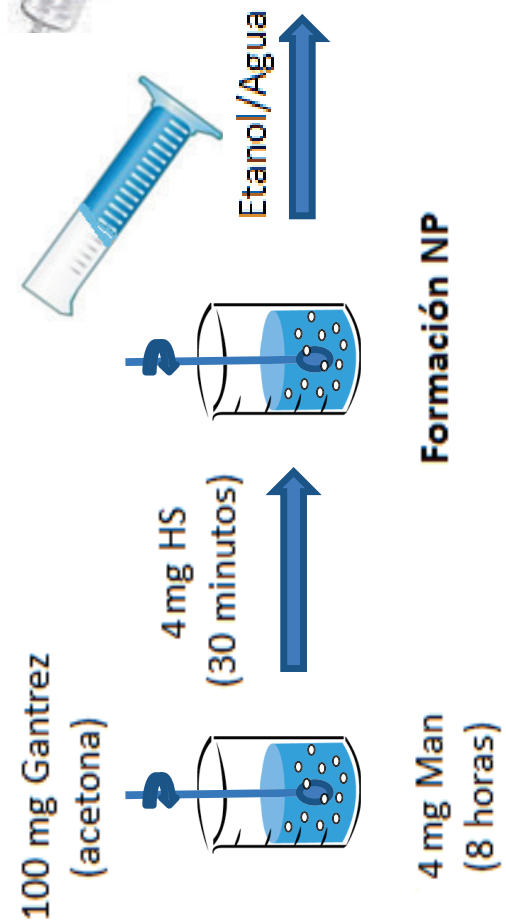


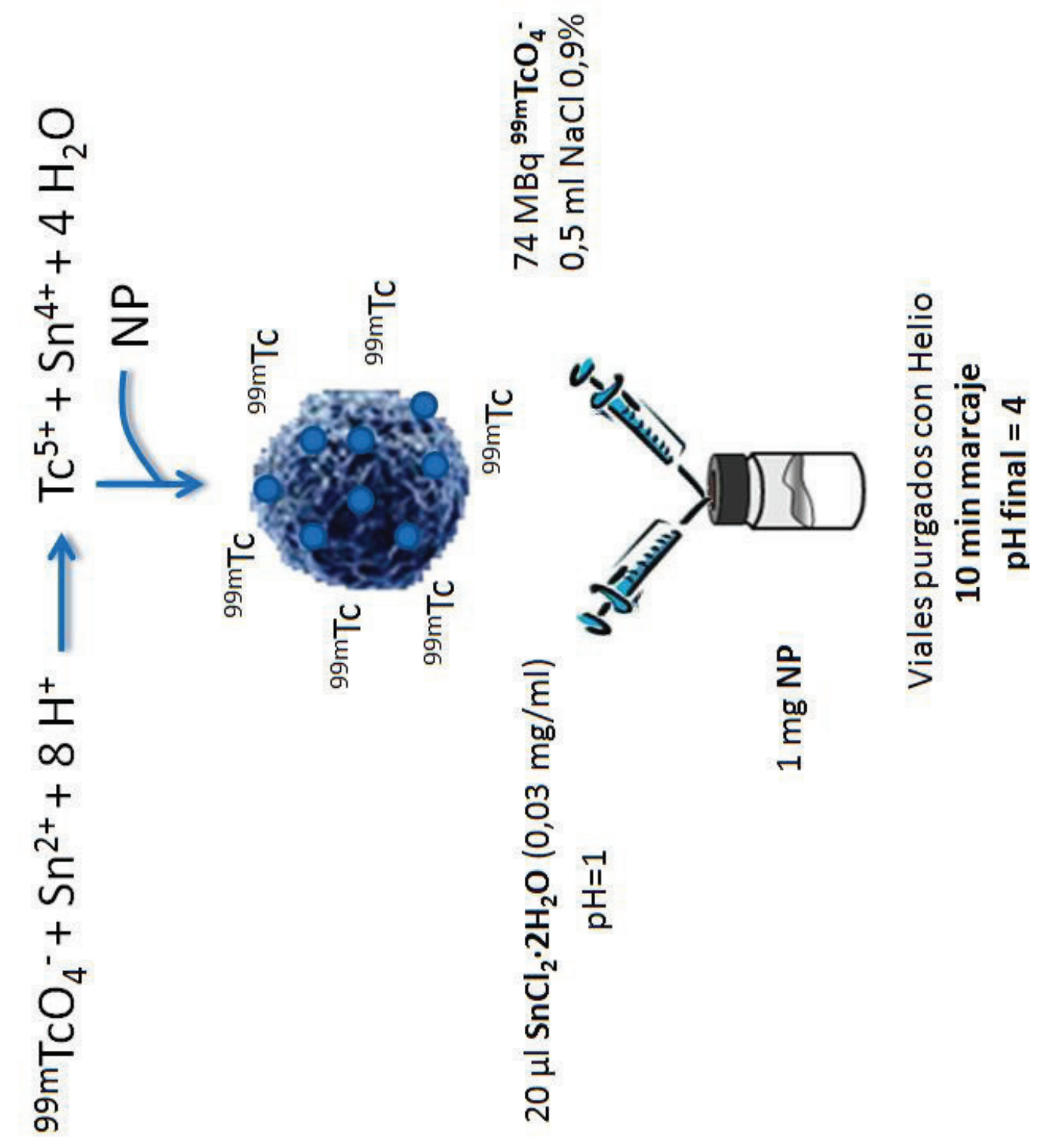




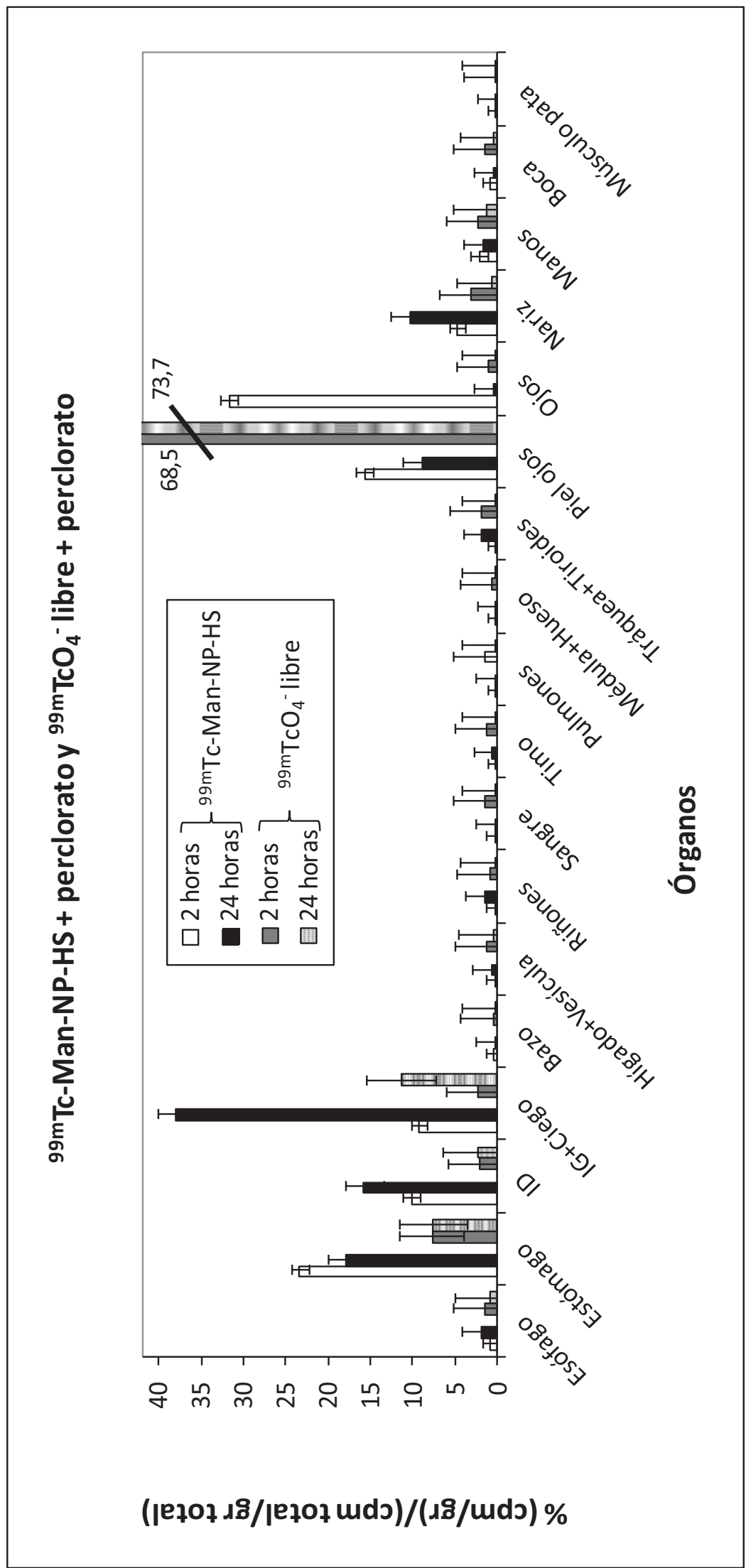



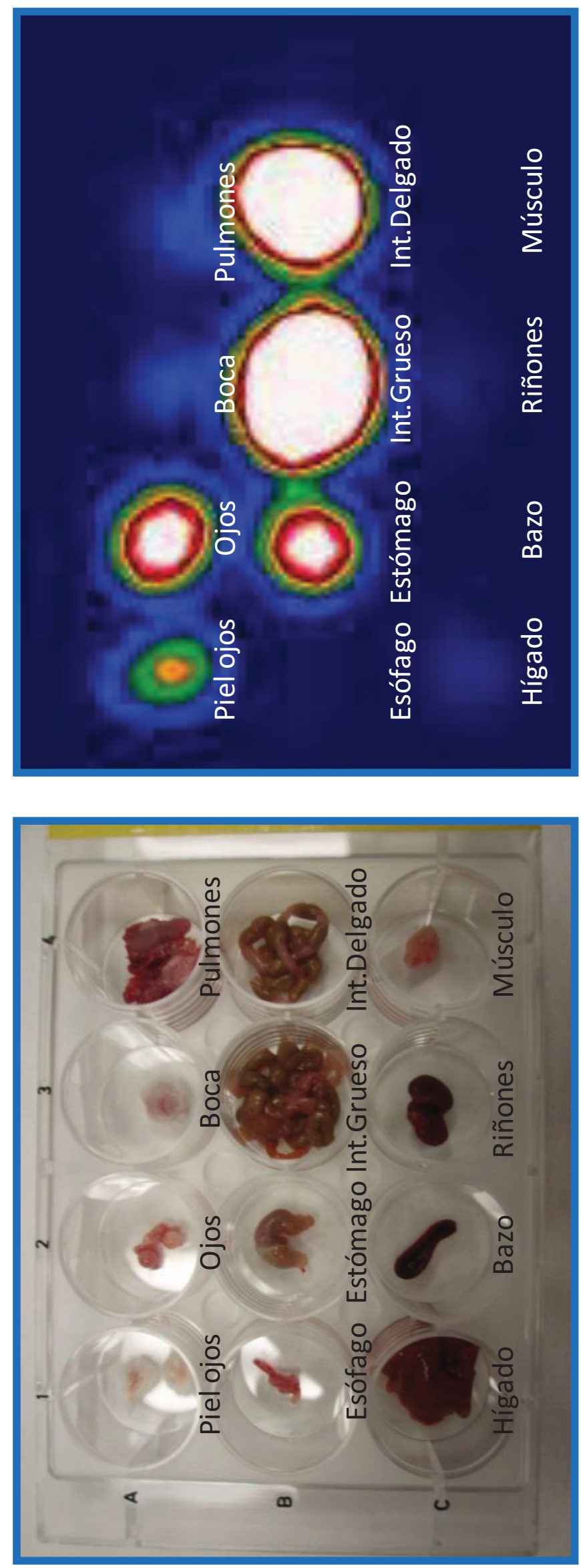

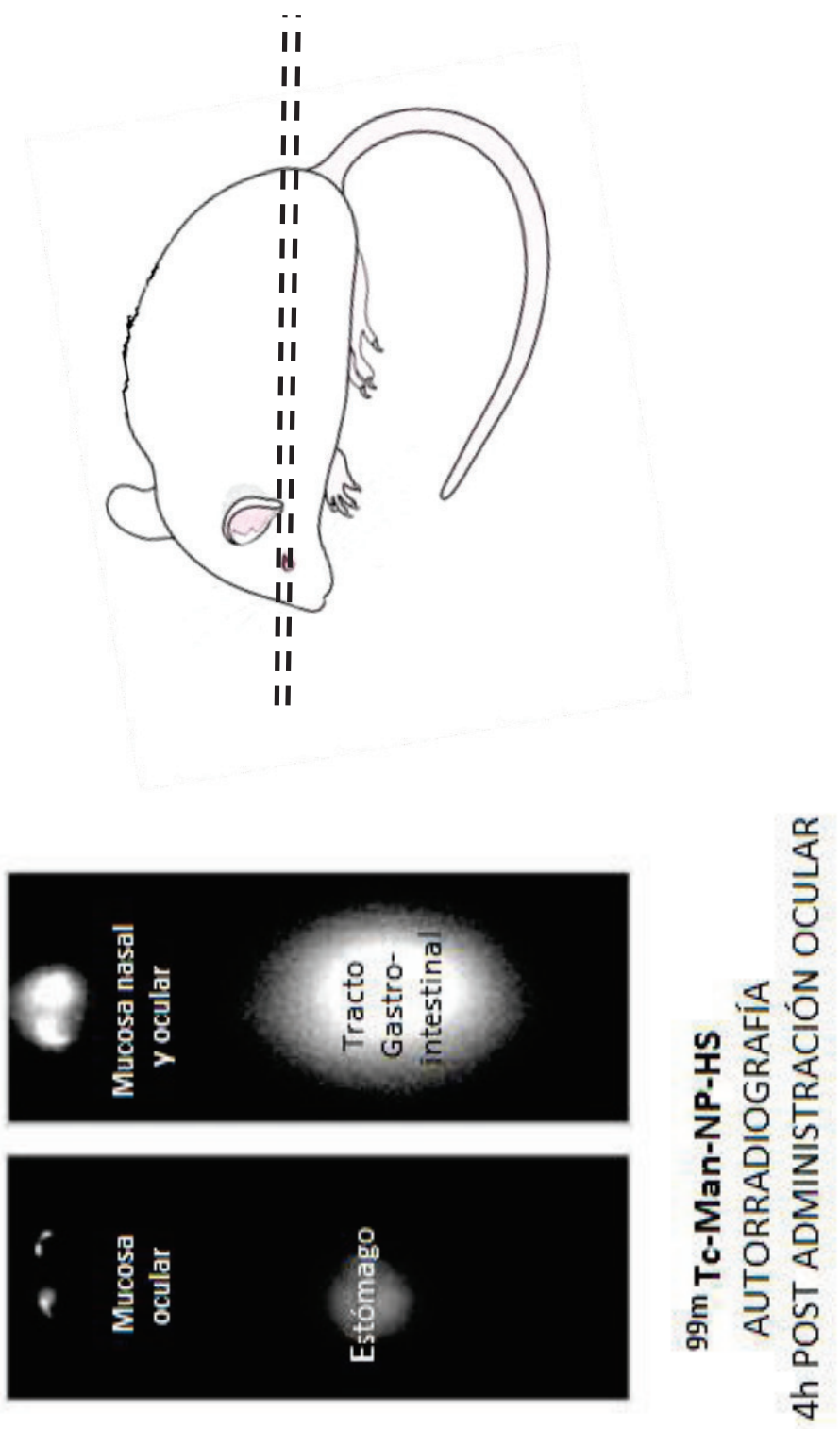